\title{
Tail distribution of the integrated Jacobi diffusion process
}

\author{
Nguyen Tien Dung ${ }^{1, *}$ and Trinh Nhu Quynh ${ }^{2}$ \\ ${ }^{1}$ Department of Mathematics, FPT University, Hoa Lac High Tech Park, Hanoi, Vietnam \\ ${ }^{2}$ Military Information Technology Institute, 17 Hoang Sam, Cau Giay, Hanoi, Vietnam
}

\begin{abstract}
In this paper, we study the distribution of the integrated Jacobi diffusion processes with Brownian noise and fractional Brownian noise. Based on techniques of Malliavin calculus, we develop a unified method to obtain explicit estimates for the tail distribution of these integrated diffusions.
\end{abstract}

Keywords Jacobi process, Tail distribution, Malliavin calculus.

AMS 2010 subject classifications 60J60, 60G22, 60H07.

DOI: $10.19139 /$ soic-2310-5070-760

\section{Introduction}

We consider Jacobi diffusion process that is defined as the solution of the scalar stochastic differential equation

$$
X_{t}=X_{0}+\int_{0}^{t}\left(a-b X_{s}\right) d s+\int_{0}^{t} c \sqrt{X_{s}\left(1-X_{s}\right)} d W_{s},
$$

where the initial conditions $X_{0} \in(0,1), a, b, c$ are positive constants and $W_{t}$ is a standard Brownian motion. It is known that this diffusion plays an important role in various fields. In population biology, it is called a Wright-Fisher diffusion with migration, see e.g. [10]. In the finance context, the Jacobi process was first used by De Jong et al. [8] to model the exchange rates in a target zone and by Delbaen and Shirakawa [3] to model interest rates. Since then, Jacobi process has been became one of popular interest rate models in finance. The main advantage of the model is that it admits lower and upper boundaries for the interest rate, hence preventing negative interest rates. Many different properties of Jacobi model can be found in the literature. Among others, we mention the works by Gouriéroux and Jasiak [6] for a multidimensional version and several applications, Ackerer et al. [2] for a new stochastic volatility model, etc.

From financial point of view, the integrated diffusion process of the form

$$
Y_{t}:=\int_{0}^{t} X_{s} d s, \quad t \in[0, T],
$$

is one of fundamental objects that needs studying, see e.g. [11] and references therein. In particular, the information about the distribution function will be very useful for applications. The reader can consult the seminal paper [4] for the results related to Cox-Ingersoll-Ross interest rate model, also see [1, 7] for other models. However, for

\footnotetext{
*Correspondence to: Nguyen Tien Dung (Email: dung_nguyentien10@yahoo.com, dungnt@fpt.edu.vn). Department of Mathematics, FPT
} University, Hoa Lac High Tech Park, Hanoi, Vietnam.

ISSN 2310-5070 (online) ISSN 2311-004X (print)

Copyright (C) 2020 International Academic Press 
Jacobi model (1), it is difficult to get such information because its volatility coefficient, $\sigma(x)=c \sqrt{x(1-x)}$, is non-Lipschitz and nonlinear.

On the other hand, in the last decades, there has been an increased interest in stochastic models based on other noise processes rather than the Brownian noise. A natural generalization of Brownian noise is fractional Brownian noise or colored noise. Recall that fractional Brownian motion (fBm) with Hurst index $H \in(0,1)$ is a centered Gaussian process $W^{H}=\left(W_{t}^{H}\right)_{t \in[0, T]}$ with covariance function:

$$
R(t, s)=\frac{1}{2}\left(|t|^{2 H}+|s|^{2 H}-|t-s|^{2 H}\right) .
$$

When $H=\frac{1}{2}, \mathrm{fBm} W^{H}$ reduces to a standard Brownian motion. The increments of the $\mathrm{fBm}$ are negatively correlated for $H<\frac{1}{2}$ and positively correlated for $H>\frac{1}{2}$. In particular, for $H>\frac{1}{2}$, fBm is a long memory process since the covariance at distance $n$ decreases as $n^{2 H-2}$ :

$$
\rho_{H}(n):=E\left(W_{1}^{H}\left(W_{n+1}^{H}-W_{n}^{H}\right)\right) \approx H(2 H-1) n^{2 H-2} \text { as } n \rightarrow \infty .
$$

The above properties, contrarily to Brownian motion, make fBm a suitable choice to model the evolution systems where the future state depends not only on the present state but also on its past states. Hence, it will be interesting to investigate fractional version of the Jacobi model. Replacing Brownian noise by fractional Brownian noise, we obtain the model

$$
X_{t}^{H}=X_{0}+\int_{0}^{t}\left(a-b X_{s}^{H}\right) d s+\int_{0}^{t} c \sqrt{X_{s}^{H}\left(1-X_{s}^{H}\right)} d W_{s}^{H}, t \in[0, T],
$$

and the integrated fractional diffusion process corresponding to (2) reads

$$
Y_{t}^{H}:=\int_{0}^{t} X_{s}^{H} d s, t \in[0, T] .
$$

Some fundamental properties of the fractional model (3), including the existence and uniqueness of solutions, were already discussed in [5]. However, the distribution of $Y_{t}^{H}$ has not been discussed yet.

Motivated by the above observations, the aim of this paper is to study the distribution of the integrated diffusions (2) and (4). Because of the complexity of stochastic calculus with respect to $\mathrm{fBm}$, it is almost impossible to compute the distribution of $Y_{t}^{H}$ explicitly. On the other hand, we would like to develop a unified method for $Y_{t}$ and $Y_{t}^{H}$. Hence, we will focus on providing the estimates for the tail distribution of $Y_{t}$ and $Y_{t}^{H}$. The main tools of the present paper are the techniques of Malliavin calculus (stochastic calculus of variations) which have been successfully used to investigate many financial models, see e.g. Chapter 6 in [12]. By using the flexible transforms, we are able to bound Malliavin derivatives of $Y_{t}$ and $Y_{t}^{H}$, and hence, we obtain explicit estimates for the tail distributions. More specifically, the main contributions of this paper are as follows

- In Theorem 3.1, we provide two explicit estimates (13) and (14) for the tail distribution of $Y_{t}$. The first one is an inequality of Gaussian type and the second one is a Bernstein-type inequality.

- Analogously, in Theorem 3.2, we also obtain two explicit estimates (19) and (20) for the tail distribution of $Y_{t}^{H}$.

The rest of the paper is organized as follows. In Section 2, we recall some elements of Malliavin calculus and a general estimate for the tail distribution of Malliavin differentiable random variables. The main results of the paper are stated and proved in Section 3. The conclusion is given in Section 4.

\section{Preliminaries}

Let us recall some elements of stochastic calculus of variations (for more details see [12]). We suppose that Brownian motion $\left(W_{t}\right)_{t \in[0, T]}$ is defined on a complete probability space $(\Omega, \mathcal{F}, \mathbb{F}, P)$, where $\mathbb{F}=\left(\mathcal{F}_{t}\right)_{t \in[0, T]}$ is 
a natural filtration generated by the Brownian motion $W$. For $h \in L^{2}[0, T]$, we denote by $W(h)$ the Wiener integral

$$
W(h)=\int_{0}^{T} h(t) d W_{t}
$$

Let $\mathcal{S}$ denote the dense subset of $L^{2}(\Omega, \mathcal{F}, P)$ consisting of smooth random variables of the form

$$
F=f\left(W\left(h_{1}\right), \ldots, W\left(h_{n}\right)\right),
$$

where $n \in \mathbb{N}, f \in C_{b}^{\infty}\left(\mathbb{R}^{n}\right), h_{1}, \ldots, h_{n} \in L^{2}[0, T]$. If $F$ has the form (5), we define its Malliavin derivative as the process $D F:=\left\{D_{t} F, t \in[0, T]\right\}$ given by

$$
D_{t} F=\sum_{k=1}^{n} \frac{\partial f}{\partial x_{k}}\left(W\left(h_{1}\right), \ldots, W\left(h_{n}\right)\right) h_{k}(t)
$$

We shall denote by $\mathbb{D}^{1,2}$ the closure of $\mathcal{S}$ with respect to the norm

$$
\|F\|_{1,2}^{2}:=E|F|^{2}+E\left[\int_{0}^{T}\left|D_{u} F\right|^{2} d u\right] .
$$

A random variable $F$ is said Malliavin differentiable if it belongs to $\mathbb{D}^{1,2}$. The next lemma comes from Corollary 4.7.4 in [14].

Lemma 2.1

Let $Z$ be a centered random variable in $\mathbb{D}^{1,2}$. Assume there exists a non-random constant $\beta$ such that

$$
\int_{0}^{T}\left(D_{r} Z\right)^{2} d r \leq \beta^{2} \text { a.s. }
$$

Then, the following estimate for tail probabilities holds

$$
P(Z \geq x) \leq e^{-\frac{x^{2}}{2 \beta^{2}}}, \quad x>0 .
$$

\section{The main results}

\subsection{Jacobi process with Brownian noise}

In this subsection, we provide two explicit estimate for the tail distribution of $Y_{t}$ defined by (2). We always assume that the parameters $a, b$ and $c$ satisfy

$$
a \geq \frac{c^{2}}{2} \text { and } b-a \geq \frac{c^{2}}{2}
$$

This assumption ensures the equation (1) has a unique solution belonging to $(0,1)$ and the boundaries $\{0\}$ and $\{1\}$ are inaccessible, see e.g. Chapter 4 in [9].

In order to be able apply Lemma 2.1, we have to prove the Malliavin differentiability of the solution to the equation (1).

Proposition 3.1

The unique solution $\left(X_{t}\right)_{t \in[0, T]}$ of the equation (1) is Malliavin differentiable and its derivative is given by

$$
D_{r} X_{t}=c \sqrt{X_{t}\left(1-X_{t}\right)} \exp \left(\int_{r}^{t} \frac{(2 a-b) X_{s}-a+c^{2} / 4}{2 X_{s}\left(1-X_{s}\right)} d s\right) \mathbf{1}_{[0, t]}(r), 0 \leq t \leq T .
$$


Proof

We first investigate the Malliavin differentiability of stochastic process $x_{t}:=\arcsin \left(2 X_{t}-1\right) \in\left(-\frac{\pi}{2}, \frac{\pi}{2}\right)$. By Itô differential formula, $x_{t}$ solves the following equation

$$
x_{t}=x_{0}+\int_{0}^{t} \frac{2 a-b-\left(b-c^{2} / 2\right) \sin x_{s}}{\cos x_{s}} d s+c W_{t}, \quad t \in[0, T] .
$$

Let us compute the directional derivative $\left\langle D x_{t}, h\right\rangle_{L^{2}[0, T]}$ for some $h \in L^{2}[0, T]$ :

$$
\left\langle D x_{t}, h\right\rangle_{L^{2}[0, T]}=\left.\frac{d x_{t}^{\varepsilon}}{d \varepsilon}\right|_{\varepsilon=0},
$$

where $x_{t}^{\varepsilon}$ solves the following equation

$$
x_{t}^{\varepsilon}=x_{0}+\int_{0}^{t} g\left(x_{s}^{\varepsilon}\right) d s+c\left(W_{t}+\varepsilon \int_{0}^{t} h_{s} d s\right), \quad t \in[0, T],
$$

where $\varepsilon \in[0,1)$ and for the simplicity, we put $g(x):=\frac{2 a-b-\left(b-c^{2} / 2\right) \sin x}{\cos x}$. We have

$$
x_{t}^{\varepsilon}-x_{t}=\int_{0}^{t}\left(g\left(x_{s}^{\varepsilon}\right)-g\left(x_{s}\right)\right) d s+c \varepsilon \int_{0}^{t} h_{s} d s, \quad t \in[0, T] .
$$

By using Taylor expansion, the above equation becomes

$$
x_{t}^{\varepsilon}-x_{t}=\int_{0}^{t} g^{\prime}\left(x_{s}+\xi_{s}\left(x_{s}^{\varepsilon}-x_{s}\right)\right)\left(x_{s}^{\varepsilon}-x_{s}\right) d s+c \varepsilon \int_{0}^{t} h_{s} d s
$$

for some random variable $\xi_{s}$ lying between 0 and 1 . The solution to (10) is given by

$$
x_{t}^{\varepsilon}-x_{t}=c \varepsilon \int_{0}^{t} h_{s} \exp \left(\int_{s}^{t} g^{\prime}\left(x_{r}+\xi_{r}\left(x_{r}^{\varepsilon}-x_{r}\right)\right) d r\right) d s, \quad t \in[0, T] .
$$

We now observe that

$$
g^{\prime}(x)=\frac{(2 a-b) \sin x-b+c^{2} / 2}{\cos ^{2} x}<0 \forall x \in\left(-\frac{\pi}{2}, \frac{\pi}{2}\right) .
$$

As a consequence, by the dominated convergence theorem, we obtain

$$
\begin{aligned}
\lim _{\varepsilon \rightarrow 0^{+}} \frac{x_{t}^{\varepsilon}-x_{t}}{\varepsilon} & =c \int_{0}^{t} h_{s} \exp \left(\int_{s}^{t} g^{\prime}\left(c_{r}\right) d r\right) d s \\
& =\left\langle h, c \exp \left(\int^{t} g^{\prime}\left(y_{r}\right) d r\right) \mathbf{1}_{[0, t]}\right\rangle_{L^{2}[0, T]}, \quad t \in[0, T],
\end{aligned}
$$

where the limit holds in $L^{2}(\Omega)$. So, by the classical results of Sugita [13], we can conclude that $x_{t} \in \mathbb{D}^{1,2}$, and its derivative is given by

$$
\begin{aligned}
D_{r} x_{t} & =c \exp \left(\int_{r}^{t} g^{\prime}\left(x_{r}\right) d r\right) \mathbf{1}_{[0, t]}(r) \\
& =c \exp \left(\int_{r}^{t} \frac{(2 a-b) X_{s}-a+c^{2} / 4}{2 X_{s}\left(1-X_{s}\right)} d s\right) \mathbb{1}_{[0, t]}(r), \quad t \in[0, T] .
\end{aligned}
$$

We now apply the chain rule of Malliavin derivatives to $X_{t}=\frac{1+\sin x_{t}}{2}$ and we obtain

$$
D_{r} X_{t}=\frac{\cos x_{t}}{2} D_{r} x_{t}=\sqrt{X_{t}\left(1-X_{t}\right)} D_{r} x_{t}, \quad t \in[0, T] .
$$

This completes the proof. 
We also need the following lemma to bound the Malliavin derivative of $\left(X_{t}\right)_{t \in[0, T]}$.

Lemma 3.1

For all $x \in(0,1)$, we have

$$
\frac{(2 a-b) x-a+c^{2} / 4}{2 x(1-x)} \leq M \leq 0
$$

where the constant $M$ is defined by

$$
M:= \begin{cases}\frac{-(2 a-b)^{2} \sqrt{\left(a-\frac{c^{2}}{4}\right)\left(b-a-\frac{c^{2}}{4}\right)}}{2\left(a-\frac{c^{2}}{4}-\sqrt{\left.\left(a-\frac{c^{2}}{4}\right)\left(b-a-\frac{c^{2}}{4}\right)\right)\left(a-b+\frac{c^{2}}{4}+\sqrt{\left(a-\frac{c^{2}}{4}\right)\left(b-a-\frac{c^{2}}{4}\right)}\right)}\right.} & \text { if } 2 a \neq b \\ -2\left(a-\frac{c^{2}}{4}\right) & \text { if } 2 a=b .\end{cases}
$$

Proof

The proof is straightforward, so we omit it. Here we only note that the function $h(x):=\frac{(2 a-b) x-a+c^{2} / 4}{2 x(1-x)}, x \in(0,1)$ attains its maximum value at $x=\frac{a-\frac{c^{2}}{4}-\sqrt{\left(a-\frac{c^{2}}{4}\right)\left(b-a-\frac{c^{2}}{4}\right)}}{2 a-b}$ if $2 a \neq b$ and at $x=\frac{1}{2}$ if $2 a=b$.

We now are in a position to state the first main results of this paper.

Theorem 3.1

Let $\left(Y_{t}\right)_{t \in[0, T]}$ be the integrated Jacobi diffusion process defined by (2). We have

I. For each $t \in[0, T]$, the expected value of $Y_{t}$ is given by

$$
\mu_{t}:=E\left[Y_{t}\right]=\left(X_{0}-\frac{a}{b}\right) \frac{1-e^{-b t}}{b}+\frac{a t}{b}, \quad t \in[0, T] .
$$

II. For each $t \in(0, T]$, the tail distribution of $Y_{t}$ satisfies

$$
P\left(Y_{t} \geq x\right) \leq \exp \left(-\frac{4 M^{3}\left(x-\mu_{t}\right)^{2}}{c^{2}\left(e^{2 M t}-4 e^{M t}+2 M t+3\right)}\right), x \geq \mu_{t},
$$

and

$$
P\left(Y_{t} \geq x\right) \leq \exp \left(-\frac{4 M^{2}\left(x-\mu_{t}\right)^{2}}{c^{2}\left(e^{2 M t}-2 M t-1\right)\left(x+\mu_{t}\right)}\right), x \geq \mu_{t} .
$$

Proof

The proof of the part I is straightforward. Indeed, we have

$$
E\left[X_{t}\right]=X_{0}+\int_{0}^{t}\left(a-b E\left[X_{s}\right]\right) d s, \quad t \in[0, T],
$$

and hence, $E\left[X_{t}\right]=\left(X_{0}-\frac{a}{b}\right) e^{-b t}+\frac{a}{b}$. So it is easy to obtain (12) because $E\left[Y_{t}\right]=\int_{0}^{t} E\left[X_{s}\right] d s$. Let us now prove the part II. By the property of Malliavin derivatives we have

$$
D_{r} Y_{t}=\int_{r}^{t} D_{r} X_{s} d s, 0 \leq r \leq t \leq T .
$$

Moreover, by the formula (8) and Lemma 3.1, we deduce

$$
0 \leq D_{r} X_{t} \leq c \sqrt{X_{t}\left(1-X_{t}\right)} e^{M(t-r)}, 0 \leq r \leq t \leq T .
$$

In order to prove (13) we observe that $\sqrt{X_{t}\left(1-X_{t}\right)} \leq \frac{1}{4}$. Hence, $D_{r} X_{t} \leq \frac{c}{2} e^{M(t-r)}$ and we obtain

$$
\left|D_{r} Y_{t}\right| \leq \frac{c}{2 M}\left(e^{M(t-r)}-1\right), 0 \leq r \leq t \leq T .
$$


Fixed $t \in(0, T]$, we consider the random variable $Z:=Y_{t}-\mu_{t}$. We have $E[Z]=0$ and

$$
\begin{aligned}
\int_{0}^{T}\left|D_{r} Z\right|^{2} d r=\int_{0}^{t}\left|D_{r} Y_{t}\right|^{2} d r & \leq \frac{c^{2}}{4 M^{2}} \int_{0}^{t}\left(e^{M(t-r)}-1\right)^{2} d r \\
& =\frac{c^{2}}{4 M^{2}}\left(\frac{e^{2 M t}-1}{2 M}-2 \frac{e^{M t}-1}{M}+t\right) \\
& =\frac{c^{2}}{8 M^{3}}\left(e^{2 M t}-4 e^{M t}+2 M t+3\right) \text { a.s. }
\end{aligned}
$$

Thus the condition (6) of Lemma 2.1 are fulfilled. Hence, we can use the estimate (7) and we obtain

$$
P\left(Y_{t}>x\right)=P\left(Z>x-\mu_{t}\right) \leq \exp \left(-\frac{4 M^{3}\left(x-\mu_{t}\right)^{2}}{c^{2}\left(e^{2 M t}-4 e^{M t}+2 M t+3\right)}\right), x \geq \mu_{t} .
$$

So (13) is verified. It only remains prove (14). We put $\bar{Z}:=\sqrt{Y_{t}}-E\left[\sqrt{Y_{t}}\right]$ and use the chain rule for Malliavin derivatives to get

$$
D_{r} \bar{Z}=D_{r} \sqrt{Y_{t}}=\frac{\int_{r}^{t} D_{r} X_{s} d s}{2 \sqrt{Y_{t}}}, 0 \leq r \leq t \leq T .
$$

This, together with (15), gives us

$$
\left|D_{r} \bar{Z}\right| \leq \frac{c \int_{r}^{t} \sqrt{X_{s}\left(1-X_{s}\right)} e^{M(s-r)} d s}{2 \sqrt{Y_{t}}} \leq \frac{c \int_{r}^{t} \sqrt{X_{s}} e^{M(s-r)} d s}{2 \sqrt{Y_{t}}}, 0 \leq r \leq t \leq T .
$$

By the Hölder inequality we deduce

$$
\begin{aligned}
\left|D_{r} \bar{Z}\right|^{2} & \leq \frac{c^{2}\left(\int_{r}^{t} X_{s} d s\right)\left(\int_{r}^{t} e^{2 M(s-r)} d s\right)}{4 Y_{t}} \\
& \leq \frac{c^{2}\left(\int_{0}^{t} X_{s} d s\right)\left(\frac{e^{2 M(t-r)}-1}{2 M}\right)}{4 Y_{t}} \\
& =\frac{c^{2}}{8 M}\left(e^{2 M(t-r)}-1\right), 0 \leq r \leq t \leq T
\end{aligned}
$$

We therefore obtain

$$
\begin{aligned}
\int_{0}^{T}\left|D_{r} \bar{Z}\right|^{2} d r & \leq \frac{c^{2}}{8 M} \int_{0}^{t}\left(e^{2 M(t-r)}-1\right) d r \\
& =\frac{c^{2}}{8 M}\left(\frac{e^{2 M t}-1}{2 M}-t\right) \\
& =\frac{c^{2}}{16 M^{2}}\left(e^{2 M t}-2 M t-1\right) \text { a.s. }
\end{aligned}
$$

which points out that the random variable $\bar{Z}$ also fulfills the condition (6) of Lemma 2.1. Hence, the estimate (7) and the fact that $E\left[\sqrt{Y_{t}}\right] \leq \sqrt{E\left[Y_{t}\right]}=\sqrt{\mu_{t}}$ give us the following

$$
\begin{aligned}
P\left(Y_{t}>x\right) & =P\left(\sqrt{Y_{t}}-E\left[\sqrt{Y_{t}}\right]>\sqrt{x}-E\left[\sqrt{Y_{t}}\right]\right) \\
& =P\left(\bar{Z}>\sqrt{x}-E\left[\sqrt{Y_{t}}\right]\right) \\
& \leq P\left(\bar{Z}>\sqrt{x}-\sqrt{\mu_{t}}\right) \\
& \leq \exp \left(-\frac{8 M^{2}\left(\sqrt{x}-\sqrt{\mu_{t}}\right)^{2}}{c^{2}\left(e^{2 M t}-2 M t-1\right)}\right), x \geq \mu_{t} .
\end{aligned}
$$


So we obtain (14) because $\left(\sqrt{x}-\sqrt{\mu_{t}}\right)^{2}=\frac{\left(x-\mu_{t}\right)^{2}}{\left(\sqrt{x}+\sqrt{\mu_{t}}\right)^{2}} \geq \frac{\left(x-\mu_{t}\right)^{2}}{2\left(x+\mu_{t}\right)}$. This completes the proof.

Remark 3.1. Clearly, when fixed $t>0$, we have

$$
\frac{4 M^{3}\left(x-\mu_{t}\right)^{2}}{c^{2}\left(e^{2 M t}-4 e^{M t}+2 M t+3\right)} \geq \frac{4 M^{2}\left(x-\mu_{t}\right)^{2}}{c^{2}\left(e^{2 M t}-2 M t-1\right)\left(x+\mu_{t}\right)} \text { as } x \rightarrow \infty .
$$

Hence, the bound (13) is better than (14) when $x$ is large sufficiently. However, when $t \rightarrow 0^{+}$, we have $\mu_{t} \simeq X_{0} t$ and

$$
e^{2 M t}-4 e^{M t}+2 M t+3=O\left(t^{2}\right), e^{2 M t}-2 M t-1=O\left(t^{2}\right) .
$$

Hence, the bound (14) will give us a better estimate if $t \simeq 0$ and $x \simeq \mu_{t}$. That is why we provided both bounds (13) and (14) as in Theorem 3.1.

\subsection{Jacobi process with fractional Brownian noise}

In this subsection, we investigate the tail distribution of $Y_{t}^{H}$ defined by (4). Following the results obtained in [5], we always assume that

$$
a<b \text { and } H \in\left(\frac{1}{2}, 1\right) .
$$

This assumption ensures that the equation (3) has a unique solution and the fractional stochastic integral $\int_{0}^{t} c \sqrt{X_{s}^{H}\left(1-X_{s}^{H}\right)} d W_{s}^{H}$ is well defined as a pathwise Riemann-Stieltjes integral (see [15] for a detailed presentation of this integral). We recall that $\mathrm{fBm} W^{H}$ admits the so-called Volterra representation (see e.g. [12] pp. 277-279)

where the kernel $K_{H}$ is given by

$$
B_{t}^{H}=\int_{0}^{t} K_{H}(t, s) d W_{s}
$$

$$
K_{H}(t, s):=c_{H} s^{1 / 2-H} \int_{s}^{t}(u-s)^{H-\frac{3}{2}} u^{H-1 / 2} d u, \quad s \leq t
$$

and $c_{H}=\sqrt{\frac{H(2 H-1)}{\beta(2-2 H, H-1 / 2)}}$, where $\beta$ is the Beta function.

Theorem 2.1 in [5] provides the following which is similar to Proposition 3.1.

Proposition 3.2

Assume that $0<a<b$ and the initial condition $X_{0} \in[0,1]$. Then the Jacobi equation (3) has a unique solution in $C^{H^{-}}[0, T]:=\bigcap_{\beta<H} C^{\beta}[0, T]$. Moreover, this solution belongs to $(0,1)$ and is Malliavin differentiable with

$$
D_{r} X_{t}^{H}=c \sqrt{X_{t}^{H}\left(1-X_{t}^{H}\right)} \int_{r}^{t} \partial_{1} K(v, r) e^{\int_{v}^{t} \frac{(2 a-b) X_{u}^{H}-a}{2 X_{u}^{H}\left(1-X_{u}^{H}\right)} d u} d v, r \leq t,
$$

where $\partial_{1} K(v, r):=\frac{\partial}{\partial v} K(v, r)=c_{H}(v-r)^{H-\frac{3}{2}} v^{H-1 / 2} r^{1 / 2-H}$.

We also have a similar estimate to that obtained in Lemma 3.1.

Lemma 3.2

For all $x \in(0,1)$, we have

$$
\frac{(2 a-b) x-a}{2 x(1-x)} \leq M^{H} \leq 0
$$

where the constant $M^{H}$ is defined by

$$
M^{H}:= \begin{cases}\frac{-(2 a-b)^{2} \sqrt{a(b-a)}}{2(a-\sqrt{a(b-a)})(a-b+\sqrt{a(b-a)})} & \text { if } 2 a \neq b \\ -2 a & \text { if } 2 a=b .\end{cases}
$$


We now note that, unlike the case of Brownian noises, the expected value of $Y_{t}^{H}$ is not easy to compute explicitly. This is due to the fact that the expectation of fractional stochastic integrals is non zero. In the next proposition, we gives an estimate for $E\left[Y_{t}^{H}\right]$.

Proposition 3.3

We have, for $0 \leq t \leq T$,

$$
E\left[X_{t}^{H}\right] \leq x_{t}^{H}:=X_{0} e^{-\int_{0}^{t} B_{s} d s}+\int_{0}^{t} A_{s} e^{-\int_{s}^{t} B_{u} d u} d s,
$$

where, for $\alpha_{H}:=H(2 H-1)$, the functions $A_{s}, B_{s}$ are defined by

$$
A_{s}:=a+\frac{c^{2} \alpha_{H}}{2} \int_{0}^{s}(s-r)^{2 H-2} e^{M^{H}(s-r)} d r, \quad B_{s}:=b+c^{2} \alpha_{H} \int_{0}^{s}(s-r)^{2 H-2} e^{M^{H}(s-r)} d r .
$$

As a consequence,

$$
E\left[Y_{t}^{H}\right] \leq \mu_{t}^{H}:=\int_{0}^{t} x_{s}^{H} d s
$$

Proof

We have

$$
E\left[X_{t}^{H}\right]=X_{0}+\int_{0}^{t}\left(a-b E\left[X_{s}^{H}\right]\right) d s+c E\left[\int_{0}^{t} \sqrt{X_{s}^{H}\left(1-X_{s}^{H}\right)} d W_{s}^{H}\right], t \in[0, T] .
$$

Denote by $D_{r}^{H} X_{t}^{H}$ the Malliavin derivative of $X_{t}^{H}$ with respect to fractional Brownian motion $W^{H}$. Then, by the transfer principle (see Proposition 5.2.1 in [12]), it follows from (16) that

$$
D_{r}^{H} X_{t}^{H}=c \sqrt{X_{t}^{H}\left(1-X_{t}^{H}\right)} \exp \left(\int_{r}^{t} \frac{(2 a-b) X_{s}^{H}-a}{2 X_{s}^{H}\left(1-X_{s}^{H}\right)} d s\right) \mathbb{1}_{[0, t]}(r), 0 \leq t \leq T .
$$

We therefore obtain

$$
D_{r}^{H} X_{t}^{H} \leq c \sqrt{X_{t}^{H}\left(1-X_{t}^{H}\right)} e^{M^{H}(t-r)}, 0 \leq r \leq t \leq T .
$$

Thanks to Proposition 5.2.3 in [12], we have

$$
\begin{aligned}
E\left[\int_{0}^{t} \sqrt{X_{s}^{H}\left(1-X_{s}^{H}\right)} d W_{s}^{H}\right] & =\alpha_{H} E\left[\int_{0}^{t} \int_{0}^{t} D_{r}^{H} \sqrt{X_{s}^{H}\left(1-X_{s}^{H}\right)}|s-r|^{2 H-2} d r d s\right] \\
& =\alpha_{H} E\left[\int_{0}^{t} \int_{0}^{s} \frac{\left(1-2 X_{s}\right) D_{r}^{H} X_{s}}{2 \sqrt{X_{s}^{H}\left(1-X_{s}^{H}\right)}}(s-r)^{2 H-2} d r d s\right] \\
& \leq \frac{c \alpha_{H}}{2} E\left[\int_{0}^{t}\left(1-2 X_{s}\right) \int_{0}^{s}(s-r)^{2 H-2} e^{M^{H}(s-r)} d r d s\right] .
\end{aligned}
$$

Inserting this relation into (18) yields

$$
E\left[X_{t}^{H}\right] \leq X_{0}+\int_{0}^{t}\left(A_{s}-B_{s} E\left[X_{s}^{H}\right]\right) d s, 0 \leq t \leq T .
$$

By Comparison theorem for differential equations we have $E\left[X_{t}^{H}\right] \leq x_{t}^{H}$, where $x_{t}^{H}$ solves the equation

$$
x_{t}^{H}=X_{0}+\int_{0}^{t}\left(A_{s}-B_{s} x_{s}^{H}\right) d s, 0 \leq t \leq T .
$$

The above equation is a linear ordinary differential equation and its solution is given by

$$
x_{t}^{H}=X_{0} e^{-\int_{0}^{t} B_{s} d s}+\int_{0}^{t} A_{s} e^{-\int_{s}^{t} B_{u} d u} d s, 0 \leq t \leq T .
$$

So we can finish the proof. 
The next statement is the second main result of the present paper.

\section{Theorem 3.2}

Let $\left(Y_{t}^{H}\right)_{t \in[0, T]}$ be the integrated fractional Jacobi diffusion process defined by (4). For each $t \in(0, T]$, the tail distribution of $Y_{t}^{H}$ satisfies

$$
P\left(Y_{t}^{H}>x\right) \leq \exp \left(-\frac{4\left(M^{H}\right)^{2}\left(x-\mu_{t}^{H}\right)^{2}}{c^{2} \int_{0}^{t} K^{2}(t, r)\left(e^{M^{H}(t-r)}-1\right)^{2} d r}\right), x \geq \mu_{t}^{H}
$$

and

$$
P\left(Y_{t}^{H}>x\right) \leq \exp \left(-\frac{2 M^{H}\left(x-\mu_{t}^{H}\right)^{2}}{c^{2} \int_{0}^{t} K^{2}(t, r)\left(e^{2 M^{H}(t-r)}-1\right) d r\left(x+\mu_{t}^{H}\right)}\right), x \geq \mu_{t}^{H} .
$$

Proof

From the derivative formula (16) and Lemma 3.2 we deduce

$$
\begin{aligned}
0 \leq D_{r} X_{t}^{H} & \leq c \sqrt{X_{t}^{H}\left(1-X_{t}^{H}\right)} \int_{r}^{t} \partial_{1} K(v, r) e^{M^{H}(t-v)} d v \\
& =c \sqrt{X_{t}^{H}\left(1-X_{t}^{H}\right)}\left(K(t, r)+M^{H} \int_{r}^{t} K(v, r) e^{M^{H}(t-v)} d v\right), 0 \leq r \leq t \leq T .
\end{aligned}
$$

Fixed $t \in(0, T]$, we put $Z^{H}:=Y_{t}^{H}-E\left[Y_{t}^{H}\right]$. Because $X_{t}^{H}\left(1-X_{t}^{H}\right) \leq \frac{1}{4}$, we obtain

$$
\begin{aligned}
\left|D_{r} Z^{H}\right| & =\left|D_{r} Y_{t}^{H}\right|=\int_{r}^{t} D_{r} X_{s}^{H} d s \\
& \leq \frac{c}{2} \int_{r}^{t}\left(K(s, r)+M^{H} \int_{r}^{s} K(v, r) e^{M^{H}(s-v)} d v\right) d s \\
& =\frac{c}{2}\left(\int_{r}^{t} K(s, r) d s+\int_{r}^{t} K(v, r)\left(e^{M^{H}(t-v)}-1\right) d v\right) \\
& =\frac{c}{2} \int_{r}^{t} K(v, r) e^{M^{H}(t-v)} d v, 0 \leq r \leq t \leq T .
\end{aligned}
$$

Since the function $v \rightarrow K(v, r)$ is non-decreasing, this implies

$$
\left|D_{r} Z^{H}\right| \leq \frac{c}{2 M^{H}} K(t, r)\left(e^{M^{H}(t-r)}-1\right), 0 \leq r \leq t \leq T,
$$

and hence,

$$
\int_{0}^{T}\left|D_{r} Z^{H}\right| d r \leq \frac{c^{2}}{4\left(M^{H}\right)^{2}} \int_{0}^{t} K^{2}(t, r)\left(e^{M^{H}(t-r)}-1\right)^{2} d r, \text { a.s. }
$$

Applying Lemma 2.1 we obtain the estimate (19). Indeed, we have

$$
\begin{aligned}
P\left(Y_{t}^{H}>x\right) & =P\left(Z^{H}>x-E\left[Y_{t}^{H}\right]\right) \\
& \leq P\left(Z^{H}>x-\mu_{t}^{H}\right) \\
& \leq \exp \left(-\frac{4\left(M^{H}\right)^{2}\left(x-\mu_{t}^{H}\right)^{2}}{c^{2} \int_{0}^{t} K^{2}(t, r)\left(e^{M^{H}(t-r)}-1\right)^{2} d r}\right), x \geq \mu_{t}^{H} .
\end{aligned}
$$


To verify the estimate (20), we consider the random variable $\overline{Z^{H}}:=\sqrt{Y_{t}^{H}}-E\left[\sqrt{Y_{t}^{H}}\right]$. We have

$$
\begin{aligned}
\left|D_{r} \overline{Z^{H}}\right| & =\frac{\int_{r}^{t} D_{r} X_{s}^{H} d s}{2 \sqrt{Y_{t}^{H}}} \\
& \leq \frac{c \int_{r}^{t} \sqrt{X_{s}^{H}\left(1-X_{s}^{H}\right)}\left(\int_{r}^{s} \partial_{1} K(v, r) e^{M^{H}(s-v)} d v\right) d s}{2 \sqrt{Y_{t}^{H}}} \\
& \leq \frac{c \int_{r}^{t} \sqrt{X_{s}^{H}}\left(\int_{r}^{s} \partial_{1} K(v, r) e^{M^{H}(s-v)} d v\right) d s}{2 \sqrt{Y_{t}^{H}}}, 0 \leq r \leq t \leq T,
\end{aligned}
$$

which, by the Hölder inequality, leads us to

$$
\left|D_{r} \overline{Z^{H}}\right|^{2} \leq \frac{c^{2}}{4} \int_{r}^{t}\left(\int_{r}^{s} \partial_{1} K(v, r) e^{M^{H}(s-v)} d v\right)^{2} d s, 0 \leq r \leq t \leq T .
$$

Once again, we use the Hölder inequality to get

$$
\begin{aligned}
\left|D_{r} \overline{Z^{H}}\right|^{2} & \leq \frac{c^{2}}{4} \int_{r}^{t}\left(\int_{r}^{s} \partial_{1} K(v, r) d v\right)\left(\int_{r}^{s} \partial_{1} K(v, r) e^{2 M^{H}(s-v)} d v\right) d s \\
& =\frac{c^{2}}{4} \int_{r}^{t} K(s, r)\left(K(s, r)+2 M^{H} \int_{r}^{s} K(v, r) e^{2 M^{H}(s-v)} d v\right) d s \\
& \leq \frac{c^{2}}{4} K(t, r) \int_{r}^{t}\left(K(s, r)+2 M^{H} \int_{r}^{s} K(v, r) e^{2 M^{H}(s-v)} d v\right) d s \\
& =\frac{c^{2}}{4} K(t, r)\left(\int_{r}^{t} K(s, r) d s+\int_{r}^{t} K(v, r)\left(e^{2 M^{H}(t-v)}-1\right) d v\right) \\
& =\frac{c^{2}}{4} K(t, r)\left(\int_{r}^{t} K(v, r) e^{2 M^{H}(t-v)} d v\right) \\
& =\frac{c^{2}}{8 M^{H}} K^{2}(t, r)\left(e^{2 M^{H}(t-r)}-1\right), 0 \leq r \leq t \leq T .
\end{aligned}
$$

We therefore obtain

$$
\int_{0}^{T}\left|D_{r} \overline{Z^{H}}\right|^{2} d r \leq \frac{c^{2}}{8 M^{H}} \int_{0}^{t} K^{2}(t, r)\left(e^{2 M^{H}(t-r)}-1\right) d r \text { a.s. }
$$

Thus the random variable $\overline{Z^{H}}$ also fulfills the condition (6), and hence, the estimate (7) gives us

$$
\begin{aligned}
P\left(Y_{t}^{H}>x\right) & =P\left(\overline{Z^{H}}>\sqrt{x}-E\left[\sqrt{Y_{t}^{H}}\right]\right) \\
& \leq P\left(\overline{Z^{H}}>\sqrt{x}-\sqrt{\mu_{t}^{H}}\right) \\
& \leq \exp \left(-\frac{4 M^{H}\left(\sqrt{x}-\sqrt{\mu_{t}^{H}}\right)^{2}}{c^{2} \int_{0}^{t} K^{2}(t, r)\left(e^{2 M^{H}(t-r)}-1\right) d r}\right) \\
& \leq \exp \left(-\frac{2 M^{H}\left(x-\mu_{t}^{H}\right)^{2}}{c^{2} \int_{0}^{t} K^{2}(t, r)\left(e^{2 M^{H}(t-r)}-1\right) d r\left(x+\mu_{t}^{H}\right)}\right), x \geq \mu_{t}^{H} .
\end{aligned}
$$

So the claim (20) is proved. The proof of Theorem is complete.

Remark 3.2. We also have the same comparison as in Remark 3.1. In fact, we can verify that the bound (19) is better than the bound (20) when $x$ is large sufficiently. Inversely, (20) is better than (19) when $t \simeq 0$ and $x \simeq \mu_{t}^{H}$. 


\section{Conclusion}

In this paper, we used the techniques of Malliavin calculus to investigate the tail distribution of the integrated Jacobi diffusion with Brownian noise and fractional Brownian noise. Our contribution is that we are able to develop a unified method to obtain explicit estimates for the tail distributions. Our work provides one more fundamental property of Jacobi models. In this sense, we partly enrich the knowledge of Jacobi models.

\section{Acknowledgments}

This research was funded by Vietnam National Foundation for Science and Technology Development (NAFOSTED) under grant number 101.03-2019.08.

\section{REFERENCES}

1. E.A. Abderrahim, E.H. Mostafa, M.G.Z.E. Abidine, Optimality of reinsurance treaties under a mean-ruin probability criterion. Stat. Optim. Inf. Comput. Vol. 7, June 2019, pp 383-393.

2. D. Ackerer, D. Filipović, S. Pulido, The Jacobi stochastic volatility model. Finance Stoch. 22 (2018), no. 3, 667-700.

3. F. Delbaen, H. Shirakawa, An interest rate model with upper and lower bounds. Asia Pac Fin Mark 9(3) (2002), 191-209.

4. D. Dufresne, The integrated square-root process. Working paper, 2001, University of Montreal.

5. N.T. Dung, Jacobi processes driven by fractional Brownian motion. Taiwanese J. Math. 18 (2014), no. 3, 835-848.

6. C. Gouriéroux, J. Jasiak, Multivariate Jacobi process with application to smooth transitions. J Econ 131(1-2) (2006), 475-505.

7. M.M. Hossain, Probability modeling of monthly maximum sustained wind speed in Bangladesh. Stat. Optim. Inf. Comput. Vol. 7 , March 2019, pp 75-84.

8. F. De Jong, F.C. Drost, B.J.M. Werker, A jump-diffusion model for exchange rates in a target zone. Statistica Neerlandica, 55 (2001), $270-300$.

9. A. Kaplun, Bounded short-rate models with Ehrenfest and Jacobi processes. Ph.D. thesis, Technical University of Dortmund, 2010.

10. S. Karlin, H.M. Taylor, A second course in stochastic processes. Academic Press, New York (1981).

11. M. Li, Derivatives pricing on integrated diffusion processes: a general perturbation approach. The Journal of Futures Markets, 35(6) (2015), 582-595.

12. D. Nualart, The Malliavin Calculus and Related Topics. 2nd edition, Springer 2006.

13. H. Sugita, On a characterization of the Sobolev spaces over an abstract Wiener space. J. Math. Kyoto Univ. 25 (1985), no. 4, 717-725.

14. N. Privault, Stochastic analysis in discrete and continuous settings with normal martingales. Lecture Notes in Mathematics, 1982. Springer-Verlag, Berlin, 2009.

15. M. Zähle, Integration with respect to fractal functions and stochastic calculus. Part I, Probab. Theory Related Fields 111 (1998), 333-374. 